\title{
PRINCIPES ÉTHIQUES A PRIORI ET JUSTIFICATIONS A POSTERIORI
}

\author{
Monica HEINTZ \\ Université Paris Ouest Nanterre La Défense, \\ Laboratoire d'ethnologie et de sociologie comparative \\ 21 allée de l'Université - 92001 Nanterre cedex \\ monica.heintz@free.fr
}

Si l'être humain dans son intégralité biologique est le même partout, comment concevoir que les principes éthiques des soins qui lui sont prodigués puissent être différents? Car si c'est une chose d'affirmer la possibilité d'une pluralité des points de vue ou des valeurs, c'en est une autre de les accepter et de mettre en place des mécanismes sociaux permettant d'en prendre compte. Nous sommes aujourd'hui en Occident témoins d'une prise de conscience grandissante de la relativité de nos valeurs morales. Entre autres, le caractère universel de la bioéthique, auparavant considérée comme naturellement dérivée des progrès de la science, se trouve remis en question. Les anthropologues admettent que la diversité des cultures va de pair avec une diversité des principes moraux. Mais il n'en va pas de même de la diversité des valeurs morales et de la diversité des habitudes culinaires, bien que ces points de vue normatifs différents se disputent le corps et la psyché humaine. L'implication émotionnelle agit plus radicalement dans le premier cas: on ne peut admettre aussi sereinement qu 'un soignant tue pour éviter la souffrance d'un patient (euthanasie) qu' on pourrait accepter qu'un soignant impose un régime végétarien à son patient. Et quand la diversité culturelle existe au sein d'une société 
qui encourage le multiculturalisme, permettant aux individus de positionner leurs actions en référence à des cadres culturels et moraux différents, la scène est ouverte aux conflits menaçant l'ordre institutionnel établi pour prodiguer les soins. Les recherches des anthropologues se retrouvent alors situées sur cette frontière fine qui distingue le descriptif (l'empirique) du normatif (le pragmatique).

Après avoir interrogé les positions épistémologiques que l'anthropologue doit considérer avant d'entamer un travail empirique, nous aborderons quelques-unes des difficultés de recherche sur le terrain de l'anthropologie de l'éthique, qui sont particulièrement sensibles dans le domaine de l'éthique des soins. D'abord, il est difficile de déterminer par l'observation quels sont les cadres moraux de référence d'un acteur social. On pourrait se diriger alors vers le discours de l'acteur, mais est-il toujours en mesure d'expliquer son propre positionnement? Comment déceler, à travers la prise de parole qui suit et justifie l'action, le cadre moral qui l'anime et auquel il faut se référer pour comprendre ses actions futures? Finalement, comment savoir si une action dérive d'un autre cadre moral ou s'il s'agit d'une déviance par rapport à celui qui est en place ? Dans la plupart des sociétés où plusieurs systèmes éthiques coexistent, il y a une hiérarchisation non avouée (résultante d'un rapport de pouvoir) qui fausse les justifications a posteriori des acteurs. De plus, les anthropologues envisagent encore difficilement que le comportement individuel ne résulte pas d'un ensemble de principes moraux mis en pratique, mais d'une négociation permanente entre les cadres moraux disponibles dans la société tels qu'ils sont incarnés dans les institutions, les lois et leurs représentants d'un côté et les désirs et les expériences individuelles de l'autre, ainsi que des interactions et des délibérations entre individus.

Cette réticence est certainement due à la difficulté de rendre compte d'une complexité et d'un dynamisme dont le caractère systémique nous échappe et de considérer que l'impact de l'expérience individuelle sur la manière de recevoir ou de prodiguer des soins pourrait être faiblemen (Tié à une référence à un système moral, même si cette référence appara ns lajustification a posteriori de l'action. Elle est pourtant déterminante dans T'action, puisque l'être humain prend des raccourcis cognitifs pour résoudre un problème sur la base des solutions apprises antérieurement- cette heuristique du jugement a été décrite depuis les années 70 par par Tversky et Kahneman (Kahneman, 2011). De plus, le modèle explicatif se complexifie par l'introduction de l'inévitable dimension diachronique: les configurations et les valeurs changent dans le temps, rendant parfois la prise de décision basée sur l'expérience antérieure trompeuse.

Dans cet article, sans procéder à une description ethnographique systématique, j'illustrerai les différentes étapes de cette réflexion méthodologique d'exemples provenant d'une enquête de terrain basée sur l'observation participante que j'ai 
conduit au sein d'une ONG moldave à financement étranger (Néerlandais). Cette recherche a été menée en 2007, en République de Moldavie, dans une ville du nord du pays et un des villages adjacents.

\section{QUELQUES PRISES DE POSITION PRÉLIMINAIRES}

Il s'agit, dans cette première partie, de délimiter le terrain d'investigation de l'anthropologie de l'éthique et de faire état de quelques interrogations générales constantes qui forment l'arrière-plan de mes recherches. J'aborderai principalement la difficulté de rester neutre et la difficulté de délimiter le terrain du jugement éthique et la pluralité des cadres éthiques dans une même société.

\section{Sur la difficulté de l'anthropologue de rester neutre}

Des récits ethnographiques témoignent que, de l'Afrique subsaharienne au Proche-Orient et jusque dans l'Asie du Sud-Est, les hommes s'assurent de la fidélité de leurs femmes de diverses façons- certains en leur interdisant physiologiquement d'éprouver le plaisir par l'excision et l'infibulation. D'autres récits font état des pratiques moins récurrentes- par exemple en Thaïlande, chez les Padaung réfugiés de Birmanie, les femmes serrent leurs cous dans des anneaux étranges seulement pour le plaisir des yeux. Et que dire de la pratique des pieds bandés qui a touché des millions de Chinoises du $10^{\mathrm{e}}$ siècle et jusqu'à la révolution de 1949? Au vingtième siècle, la médecine occidentale apprenait déjà aux ethnologues, témoins de ces coutumes, que les femmes girafes qui serraient leurs cous dans ces anneaux superposés ou que les Chinoises aux pieds bandés étaient condamnées à des handicaps et des souffrances permanents. Quant aux pratiques de l'excision et de l'infibulation, elles étaient contraires à sa condamnation de la souffrance. Face à de tels phénomènes, il n'est donc pas étonnant que le concept de relativisme culturel, le rejet de l'ethnocentrisme et la tolérance envers les différences culturelles se situaient aux limites de l'acceptabilité de l'objectivité et de la neutralité de l'anthropologue observateur, en particulier des anthropologues féministes. Dans ce contexte et devant le constat que l'être humain dans son intégralité biologique est le même partout, le problème est d'en arriver à reconnaître que les principes éthiques des soins corporels qui lui sont prodigués puissent différer selon les cultures et à accepter ces variations, tout en demeurant dans les limites de ce qui peut paraître humainement acceptable.

Comprendre et vivre le relativisme moral n'est pas tâche aisée pour un observateur qui d'une part met à distance son récit personnel afin de rester du côté de la 
science et, d'une autre part, sous l'impulsion de la répulsion et de l'outrance, veut agir. Ainsi, la première règle que se donne l'anthropologue étudiant les normes et les valeurs d'une autre société, celle de rester fidèle à son ethos scientifique et de se cantonner à la description d'un phénomène sans donner des indications normatives, reste toujours la plus difficile à tenir. Et, pour un anthropologue de l'éthique, cette question est d'autant plus sensible qu'elle remet violemment en question la déontologie même de l'anthropologue. Dans la perspective d'une éthique de la responsabilité exigeante, observer sans réagir c'est accepter et collaborer. Ainsi, «se taire» est, du point de vue d'une anthropologie militante, une «suspension de l'éthique», une faute humaine sinon professionnelle (Scheper-Hughes, 1995 :409). Cette position s'oppose directement à une approche scientifique positiviste qui exige la neutralité.

Pourtant, les considérations morales occidentales, ces protestations outrées émanant des sociétés aisées ne peuvent non plus se transformer en accusations systématiques des pratiques d'une culture entière, sans considérer son histoire, ses hommes, ses femmes et ses perspectives. Un comportement, croyance ou valeur morale collective acquièrent leur force de l'étendue de la conviction qui les soutient. Même «erronés » à nos yeux, leur caractère collectif leur donne une valeur qui s'impose à notre considération. Une tradition ne survit pas seulement par «habitude», mais aussi par conviction. Et cette conviction des autres dans leurs croyances, il est inopportun de la juger vide de sens, avant d'interroger ce qui la génère. Ainsi il est intéressant de noter la distance que l'anthropologue Saba Mahmood met aujourd'hui par rapport à ses écrits antérieurs et envers le féminisme séculaire dans la préface de son livre Politics of Piety (2005 : xi), une fois qu'elle entreprend d'étudier les mouvements piétistes en Égypte. Rester neutres, alors que malgré notre profession d'anthropologue nous sommes ancrés dans une société avec ses propres valeurs et que nous ne pouvons adhérer à plusieurs valeurs contradictoires à la fois, relève d'un pari que seul le respect profond envers les autres manifestations de la vie et de la culture humaines peut nous aider à tenir (Heintz, 2009).

\section{Sur la difficulté de délimiter le terrain du jugement éthique}

Si la volonté de relativiser les valeurs occidentales nous éloigne de la posture militantiste, il ne s'agit nullement de tomber dans le désengagement souvent imputé au relativisme culturel et moral. Le choix des problématiques de recherche anthropologique, s'il est en accord avec les préoccupations des sociétés étudiées, est en lui-même un choix éthique, puisqu'il vise à mettre en lumière des zones de contradictions et de conflits sur lesquelles ces sociétés, en étant informées des 
résultats, sauront trancher. Mais ce choix éthique de l'anthropologue, qui peut sembler minimaliste aux yeux des anthropologues militants, est aussi truffé de difficultés épistémologiques. Déterminer quelle problématique présente une valeur aux yeux de la société étudiée, est déjà le résultat d'un effort cognitif et émotionnel de la part de l'anthropologue, qui lui exige de se distancier de sa propre hiérarchie de valeurs, afin d'être capable d'identifier celle des autres. Ce, d'autant plus que ce qui est lourd de jugement moral pour nous, ne l'est pas forcément pour nos informateurs. Il est ainsi difficile d'être sensible à leurs préoccupations éthiques, alors même qu'un code de traduction entre nos valeurs et les leurs n'existent pas et que nous ignorons ce qui relève d'un choix éthique difficile pour le «radicalement autre» (Baumard et Sperber, $2007: 6$ ).

Pour essayer de résoudre ce problème épistémologique, la psychologie évolutionnaire (Baumard, 2010) essaie de rendre la traduction possible en cherchant les limites d'un noyau commun de valeurs morales inhérentes à l'être humain de par l'unité de l'espèce. Dans cette perspective, si certaines émotions et intuitions morales étaient universelles, les cadres moraux de chaque société seraient des produits culturels différents, dérivant du même noyau biologique, et ils deviendraient traduisibles de l'un à l'autre grâce à une «grammaire» morale universelle ainsi révélée. En attendant, d'ici à ce que cette hypothèse soit infirmée ou confirmée empiriquement, nous sommes obligés de contourner le problème de traduction en repérant les conjonctures moralement difficiles pour nos informateurs d'après l'investissement émotionnel qu'ils mettent à juger ce qui est acceptable et ce qui ne l'est pas. Aussi approximative que cette définition des phénomènes moralement sensibles puisse paraître, elle recentre le regard de l'anthropologue vers ce qui a de l'importance pour une société, que ce phénomène relève aux yeux de l'anthropologue du domaine des valeurs morales ou pas.

\section{Sur la pluralité des cadres éthiques propres à une société}

L'avantage d'un regard plus éloigné, s'il n'est pas simplement indifférent et désengagé, est qu'il est moins ancré politiquement, donc plus libre d'embrasser l'entièreté du phénomène social sans succomber aux effets rhétoriques des discours dominants dans la société en question. Car force est de constater que le modèle d'une société à l'écart du monde, qui disposerait d'un cadre éthique unique et inchangé, qu'il suffirait de repérer et traduire pour un lecteur occidental n'existe plus; des auteurs comme Johannes Fabian (1983) ont mis impitoyablement un terme à cet imaginaire ethnographique. Dans le monde globalisé d'aujourd'hui, les valeurs occidentales ont pénétré toutes les sociétés, à travers l'application des droits de l'homme, les ONG occidentales, les normes corporatistes des 
entreprises multinationales et le modèle que constitue le touriste en vacances. Les anthropologues montrent souvent dans leur ethnographies comment les sociétés traditionnelles se sont vues remises en question de l'intérieur par leurs propres membres catéchisés ailleurs (l'exemple du rôle des prêtres autochtones dans l'évangélisation et l'éveil éthique des Urapmin décrits par Robbins, 2004) ou par les nouveaux venus (les évangélisateurs du management à l'occidentale en Europe de l'Est vus par Dunn, 2004). Ce mouvement n'est pas à sens unique: l' «Autre» est aussi le migrant qui porte les valeurs culturelles de sa société d'origine vers la société d'accueil, surtout dans les sociétés qui encouragent le multiculturalisme (Wikan, 2002). Ainsi, la circulation des hommes et des idées, a multiplié les cadres éthiques à l'oeuvre dans chaque société en tant que cadres de références de l'action. Certains d'entre eux sont incompatibles, d'autres seulement partiellement compatibles.

Dans la rencontre entre l'Occident et «le reste du monde», le premier reste généralement crédité des «meilleures » valeurs grâce au jeu de pouvoir établi par l'hégémonie économique. Un auteur tel que Herzfeld (1987) montrent comment le rapport de subordination dans lequel se trouve celui qui a tort de ne pas avoir raison s'accompagne de honte (1987:38). Dans une confrontation de modèles éthiques, le modèle dominant est aussi considéré comme «meilleur», envoyant les autres modèles dans le tort et la déviance. Qu'en est-il alors des conventions universelles concernant les droits de l'homme, sont-elles le simple résultat d'un rapport de force inégal entre l'Occident et le reste du monde? Par exemple, la Déclaration universelle des droits de l'homme de 1948, émane de l'Occident et est applicable dans tous les pays signataires de la charte de l'ONU. Son non-respect ou des défaillances dans ses applications peut entraîner d'importantes pressions économiques et politiques. Il faudrait rappeler ici que les anthropologues américains qui participaient aux débats conduisant à son établissement s'en sont retirés suite à une prise de conscience de son ethnocentrisme (dû à son centrage exclusif sur l'individu, négligeant le groupe social), pourtant signalé à la commission dès 1947 (The Executive Board of the American Anthropological Association, 1947 : 539). La Convention relative aux droits de l'enfant de 1989 émane également de l'Occident. Elle a été implantée dans plusieurs pays du monde par des ONG détenant des ressources souvent nécessaires pour les populations ou en exerçant des pressions financières et économiques. Fait à noter, elle a été condamnée par des ONG africaines qui lui reprochent d'être inadaptée au cas des enfants africains - Howell, 2007).

A leur défense, on doit noter que les gardiens des droits de l'homme soutiennent un point de vue moral qui existe déjà dans l'autre société, mais qui est écrasé par un point de vue dominant contraire. Par exemple, des ONG telles Amnesty International, soutiennent un point de vue contraire à celui (dominant) du gouvernement ou de la majorité du pays dans lequel elles interviennent. Ces ONG arrivent 
souvent à s'implanter dans ces pays à l'aide d'une petite minorité locale (en situation de dissidence) qui partage les mêmes valeurs universalistes que l'ONG. Dans ces cas, quel est le rapport de pouvoir à dénoncer par l'anthropologue? Il est difficile pour un anthropologue occidental de ne pas adhérer au contenu de ces chartes universelles, puisqu'il y retrouve globalement ses propres valeurs. De la même façon que ces chartes, les soins biomédicaux occidentaux s'imposent dans les pays du Sud en vertu de leur «légitimité». A tort ou à raison, ceci se fait souvent en faisant fi des structures alternatives locales au risque de les écraser au détriment, mais aussi au nom du bien-être des patients (Cross and MacGregor, 2010).

Au niveau théorique, même si on soutient que le «regard éloigné» de l'anthropologue peut permettre une mise à distance des différents discours éthiques en concurrence dans une société, quant il s'agit d'appliquer cette théorie, il faut prendre conscience que l'appartenance du chercheur aux valeurs occidentales, largement légitimités de par la supériorité des pouvoirs exercés par les pays qui en font la promotion, pourrait invalider le raisonnement.

Néanmoins, même en supposant que deux cadres éthiques différents s'affrontent d'égal à égal, comment gérer ce qui peut rapidement devenir un affrontement entre deux «mondes»? Dan Justification (Boltanski et Thévénot, 1991) Boltanski et Thévénot tentent de répondre à cette question en suggérant les voies suivantes: départager le meilleur cadre éthique - i.e. le plus satisfaisant pour la majorité dans un contexte donné - par une épreuve, trouver un compromis de principe ou suspendre l'impératif de justification rationnelle - c'est-à-dire agir au cas par cas. Hors des abstractions des principes, Unni Wikan répond en faisant l'analyse approfondie du cas Fatima, un crime d'honneur ayant eu lieu dans une famille kurde en Norvège (2008). Avec la précision d'un jugement en cour (comme un jury représentant les deux univers moraux qu'elle connaît tout aussi bien), elle milite en prenant position contre le cadre moral kurde qui pousse au meurtre et qu'on excuse facilement en prétextant son fondement «traditionnel». Ainsi, laissant les réflexions autour des principes de gestion du relativisme culturel aux philosophes, des anthropologues telles que Unni Wikan essaient plutôt d'apporter des éléments de réponse en explorant les multiples facettes dévoilées par les études de terrain et qui donnent à repenser en retour les principes de gestion du relativisme.

\section{LES ACTIONS ET LES DISCOURS COMME LIEUX D'OBSERVATION DES CADRES ÉTHIQUES}

Quand on planifie un projet de recherche ou un programme de santé, par exemple, nous sommes contraints par la rationalité scientifique d'élaborer une 
méthode de recherche qui postule l'existence des discours sous forme d'énoncés de principe, des actions observées qui seront plus ou moins en conformité avec l'expression verbale de nos cadres d'action, sui 10 justifications qui rapprocheront a posteriori le principe de l'action effectivement accomplie. Mais, dans la réalité, les liens, l'ordre, la dialectique entre action et valeur sont beaucoup plus complexes que ce qu'un projet de recherche méthodiquement conçu nous permet d'envisager. Tout d'abord parce qu'un principe éthique ne se traduit pas toujours en action. L'acteur n'a pas toujours la liberté de l'y traduire ou il peut aussi arriver qu'il ne le souhaite pas. Ensuite, les justifications peuvent pousser à revoir a posteriori le contenu premier des principes, afin de les accorder à l'action ayant eu lieu ou comme résultat de l'interpersonnalité et négociabilité des valeurs morales dans l'interaction entre individus ou dans leur mise en action. C'est ce que nous tenterons de montrer dans la suite de cet article, en traitant de l' «observation» des principes éthiques, de la justifications et des réaménagements éthiques a posteriori, et des enjeux de négociations.

\section{«Observer» un principe éthique}

Les valeurs morales n'ont pas d'existence tangible. Il est donc impossible de les appréhender directement. Le chercheur tente d'extraire à partir de l'observation des actions (faits, gestes, paroles), les valeurs qui les sous-tendent. De plus, il arrive souvent que l'individu ne peut s'exprimer d'une manière explicite sur les raisons sous-tendant son geste ou il peut aussi intentionnellement en taire ces raisons. La première étape est de déterminer les phénomènes observables qui peuvent nous renseigner sur les croyances et les valeurs morales des acteurs sociaux à l'étude.

L'ensemble des discours et des pratiques relevant du domaine de l'éthique des soins est particulièrement propice pour ce type d'observation. Entre autres, parce qu'il donne souvent accès à des situations de conflit entre plusieurs cadres éthiques ou à des changements de ces cadres. Lorsqu'il est questions de soins, de maladie et de santé, même si l'objet du conflit ou les changements peuvent être techniques et sans conséquence apparente pour l'ordre des valeurs, il peut pourtant déclencher des débats et des changements de cadre éthique. Il y a quelques années, l'installation d'une salle de fitness par une ONG moldave à financement étranger dans un centre de soins externes pour les personnes âgées dans un village moldave, m'a permis d'observer ces phénomènes.

Cette ONG avait plusieurs projets d'aide aux personnes âgés: consultation et distribution de médicaments à domicile (personnel médical), centre externe de soins (animation autour de la salle de fitness), soutien psychosocial à domicile et en centre (psychologues et assistantes sociales en visite à domicile et repas dans 
le centre de soins). Un premier débat qui a animé la communauté, de même que le personnel de l'ONG, portait sur l'utilité des soins autres que médicaux, moins appréciés par les patients. Or, le centre de soins externes ne proposait une permanence médicale qu'une fois par semaine et l'essentiel de son activité se concentrait autour des repas chauds et de la salle de fitness.

C'est dans ce contexte que, en observant l'utilisation de cette salle par des femmes qui n'avaient pas fait de sport depuis leur passage à l'école une cinquantaine d'années auparavant, j'ai pu assister à la naissance de débats moraux sur le droit au bien-être, voire à la sexualité de personnes âgées, parmi les villageois plus jeunes. L'existence de ces appareils ultramodernes, lesquels paraissaient incongrus dans un cadre villageois où la nécessité de remédier à la pauvreté des personnes âgées était considérée comme étant prioritaire à l'amélioration de leur «forme» physique, fut un vecteur de changement dans la perception de l'âge, des droits, des souhaits et des désirs des personnes âgées, par eux-mêmes et par les autres.

Cette situation me fournissait un riche corpus de données à analyser et à interpréter. Il s'agissait alors de mettre discours et actions en cohérence, de les compléter par les non-dits, les intentions manquées, ainsi que par les discours et les actions dont l'incidence était indirecte. Ainsi, bousculée par un événement tel que l'introduction d'appareils considérés utiles «pour les jeunes », l'éthique quotidienne se rapportant aux personnes âgées est devenue plus facilement observable. La mise en relief plus systématique de cette éthique quotidienne, vers laquelle pointent de nouvelles recherches comme celles réunies par Michael Lambek (2010), montre combien les valeurs et les actions sociales concordent même là où on s'y attendrait le moins.

On constate aussi que l'ensemble de principes éthiques gouvernant les soins qu'on peut énoncer sous forme orale ou écrite ne constitue qu'une partie, bien qu'importante, de l'éthique des soins. C'est ce qu'on aurait pu appeler autrefois «l'ethno-éthique» appliquée au domaine de la santé, de la même façon dont on parlait alors d'ethnobiologie, ethnomusicologie, etc. C'est-à-dire, le corpus de connaissances et d'informations éthiques, biologiques ou musicologique dont disposait une société/ethnie. Toutefois, c'est par leur mise en pratique que ces corpus théoriques prennent formes et existent. Ainsi, on peut considérer qu'il est possible d'appréhender l'éthique d'un groupe autant à travers ses pratiques que dans les discours idéalisés sur ce que la pratique devrait être. Pour débusquer les valeurs qui modulent les actions en temps réel, il est plus profitable d'analyser les débats occasionnés par la salle de fitness autour de personnes âgées et des actions des jeunes autour d'eux (aide à la construction de la salle, encouragement à s'y rendre, etc.) que de conduire des entretiens génériques parmi les jeunes du village sur comment devraient être traitées les personnes âgées. 
La transposition des valeurs individuelles en pratiques est intrinsèquement liée à la liberté dont dispose l'acteur de passer à l'acte. L'observation d'une pratique/ action ne renseigne pas sur la valeur qui sous-tend l'action si elle n'est pas accompagnée de l'appréciation de la liberté de faire ce qu'on pense être le bien. Ici, l'expérience moldave vient préciser ce que «liberté» ou manque de liberté veut dire. L'initiative de l'ONG a été difficile à implanter parce qu'elle était menée par une femme adhérant à un parti politique d'opposition dans un village où le maire appartenait au parti au pouvoir. Pour cette raison, le maire s'était farouchement opposé au projet et avait usé de tous ses liens parentaux et politiques pour empêcher les jeunes d'aider à la construction du centre. Par solidarité, certains membres de sa famille ont été contraints de ne pas travailler au centre et les personnes âgées apparentées ont été contraintes de ne pas y mettre les pieds. Ainsi, les discours et les actions des ces gens ne nous ont pas permis de tirer quelque conclusion que ce soit sur l'intérêt porté au programme. Par contre, ils nous ont éclairés sur le fait que, dans ce contexte, les valeurs familiales devaient prendre le pas sur d'autres valeurs. D'ailleurs, le maire a été jusqu'à utiliser l'appartenance identitaire au village dans ses efforts d'opposition au projet de la présidente de l'ONG. «Tu n'es pas de ce village» lui disait-il.

L'observation participante permet d'identifier les conflits entre «théorie» et «pratique», entre discours et faits. Ces conflits se traduisent souvent dans le discours, en prenant la forme de raisonnements et de justifications a posteriori. Il arrive parfois que ces raisonnements et justification soient faits a priori dans les cas où les acteurs peuvent anticiper une action «déviante». Ces justifications ont comme fonction thérapeutique de réduire l'éventuelle dissonance cognitive, provoquée par l'écart entre la parole et les gestes, qui pourrait apparaitre quand l'acteur entre en conflit avec les valeurs locales malgré sa bonne foi et son souhait d'agir en conformité avec ses valeurs. Même en présence d'une tromperie intentionnelle, elles servent à dissimuler les intentions de l'acteur ou à lui donner une image favorable face à la communauté.

\section{Justifications et réaménagements éthiques a posteriori}

La dissonance cognitive est un état de fait désagréable pour l'acteur social. Celui-ci s'efforcera donc de mettre son action en accord avec son discours. Alors que l'action est irréversible, le discours peut corriger les faits ou les principes afin de rétablir l'harmonie (phénomène étudié depuis les années 50, voir Festinger et al, 1956). Le rejet de la société est également un fait désagréable pour l'individu dont l'action n'est pas conforme aux normes attendues. Le discours de justification peut alors servir à corriger le rejet social (parfois juste la mauvaise impression, la 
gêne, etc., ressenties en société) par deux moyens: enrichir et préciser les faits ou retravailler les principes en les rendant moins rigides ou plus «élastiques».

Un exemple notoire est le vol de ce qui était en fait la propriété de l'État durant l'époque socialiste. À cette époque, les concepts d' «État», de «propriétaire» ou de «peuple» était abstraits, vagues et par conséquent amalgamés. Ainsi un vol de matériel dans une usine n'était pas considéré comme tel, et après coup, les voleurs s'y référaient différemment en disant emprunter, se débarrasser de futurs déchets, etc. (Firlit and Chlopencki, 1992). Pareillement, dans une ville de Moldavie, pour ce qui concerne les soins prodigués par les enfants à leurs apparentés âgées, on pouvait s'excuser en rappelant que les voisins étaient encore moins impliqués. Ainsi, on pouvait moralement se permettre d'abaisser les exigences. Souvent la comparaison explicite se faisait par rapport au nombre de visites faites aux parents âgés en besoin.

La justification est un des moyens de se convaincre soi-même et/ou les autres du bien-fondé d'une action qui n'est pas facile à accepter d'un point de vue éthique, par soi-même, par les autres, ou par l'ensemble des individus concernés. Elle n'est pas un vecteur de communication verbale des valeurs comme il aurait été commode de penser, mais d'une portion importante, dans un corpus de données, d'événements linguistiques ou factuels révélant une forme de subjectivité éthique. En fait, la justification montre le plus souvent que l'action attendue n'a pas été accomplie, donc qu'on ne peut déduire une valeur morale à partir de son observation. Quand, dans un hôpital sous-financé de Roumanie au début des années 2000, les infirmières grondaient les mères qui n'avaient pas pris soin d'administrer les médicaments de leurs enfants pendant la nuit, justifiant leur demande par le nombre faible d'infirmières pour surveiller tout le palier, elles savaient que dans des conditions plus favorables, elles auraient dû s'en occuper elles-mêmes. Cet exemple montre qu'il n'est cepen_(20) pas toujours possible d'inférer des principes éthiques à partir des actions observees, quand tout porte à croire que ce discours de responsabilisation médicale était circonstanciel et ne reflétait ni les principes de soins de l'établissement ni ceux des infirmières.

\section{Négociations et délibérations : un modèle d'analyse interactionniste}

Un troisième élément vient aussi affaiblir le lien entre principe a priori et justification a posteriori: la négociation ou délibération avec soi-même ou les autres. Par exemple, dans un débat il est possible de changer d'avis, d'adopter le point de vue et les valeurs de l'autre. Il reste toutefois plus facile de changer l'interprétation 
d'un fait donné que de véritablement changer ses principes. Dans l'exemple donné plus haut sur l'introduction d'une salle de fitness dans un village, les plus jeunes gravitant autour du projet avaient du mal à voir comment le fait de faire du sport pourrait véritablement motiver les personnes âgées à venir au centre. Ils prédisaient même que le projet tomberait à l'eau si la distribution de nourriture qui accompagnait ces activités cessait, même si l'équipement de fitness restait sur place. Par contre, au terme de moult débats, certains finissaient par être convaincus, par l'administratrice du projet, du bonheur ressenti par leurs aînés impliqués. Toutefois, ils n'allaient pas jusqu'à adhérer à l'idée que le sport était plus susceptible d'augmenter l'espérance de vie à tout âge qu'une nourriture à base de viande. Certains critiquaient ouvertement la décision des organismes subventionnaires d'investir dans une salle de gym au lieu d'octroyer tout l'argent au financement de repas chauds.

Si les valeurs sont façonnées en interaction avec les faits, elles restent néanmoins dépendantes de certains cadres éthiques véhiculés dans la société. Il est rare que des arguments personnels et muries par les acteurs apparaissent spontanément pendant le débat. Dans les débats on utilise plus souvent des arguments tirés des médias, de l'église et d'autres sources éducationnelles, même s'ils sont interprétés par les acteurs à la lumière de l'expérience personnelle. Lors de mon expérience au sein de l'ONG moldave, réussir à identifier les cadres éthiques véhiculés dans la société auxquels les actions et les discours étaient tributaires, était un jeu intellectuel intéressant qui permettait de voir l'éventail de possibilités éthiques existantes à un certain moment dans la communauté. Mais, si j'utilise le terme «jeu», c'est pour souligner à quel point le fait de remonter de la parole de mes interlocuteurs vers leurs principes et valeurs profondes était un exercice quasi impossible.

Ce qui était observable dans les débats qui occupaient ce village de Moldavie était le manque de consistance des opinions, des valeurs et des croyances exprimées. Dans le conflit politique qui sous-tendait les tensions entre l'ONG et la mairie, les adversaires pouvaient utiliser, dans une même phrase des arguments politiques contradictoires au sujet: des critères de sélection des bénéficiaires, de l'étendu des soins prodigués, de la rémunération des soignants. Ceci était certainement dû à une inconsistance interne des discours d'emprunt, insuffisamment réfléchis et repris sur tout l'échiquier politique national, mais aussi à la superposition d'un autre agenda dont les enjeux étaient associées au pouvoir. Le refus de comprendre et de se plier aux arguments de l'autre obligeait, en cours de route, à opérer des changements d'arguments qui n'étaient dues qu'aux enjeux politiques des élections locales. Pourtant, si on étend l'observation au-delà du champ de la confrontation conflictuelle devant témoins, car ces affrontements étaient indirects et avaient lieu lors des discours publics et non pas en face à face, on constate que l'inconsistance des principes d'un même individu est plus courante qu'on serait porté à le croire. On 
pourrait ici sortir du cadre de l'explication strictement rationnelle pour introduire les émotions, les désirs et la volonté dans notre modèle explicatif, mais je propose une solution de rechange pour capter ces éléments: le détour par l'expérience.

\section{L'EXPÉRIENCE}

Nous entendons ici le mot «expérience» de deux manières: comme expérience de vie qui fait aussi introduction à l'analyse diachronique et comme expérience au sens phénoménologique.

\section{L'expérience de vie: le poids du passé}

L'approche interactionniste, dont les principes de base sont détaillés plus haut et illustrés dans les exemples choisis, est centrée sur des négociations observées dans la quasi-synchronicité des événements. Toutefois, les acteurs sociaux engagés dans ces interactions s'appuient sur leurs rencontres passées pour déterminer les arguments les plus efficaces et anticiper les intentions cachées de l'adversaire. Les émotions qu'ils ressentent et qui orientent leurs discours, ne sont pas dérivées exclusivement du moment présent. Elles surgissent aussi du passé, de l'expérience d'interactions similaires, de ce vécu qui détermine les représentions différentes que les acteurs se font des mots prononcés, des scénarios évoqués dans le débat et des intentions présumées de l'interlocuteur. Elles sont dérivées également des désirs - qui tendent vers le futur - et de la rencontre entre des contraintes objectives et l'étendue de l'imagination éthique.

Il y a un air de déjà-vu dans la plupart de nos interactions sociales. Notre cerveau fait alors l'économie de prendre le raccourci du «déjà pensé » et du «déjà jugé», sans faire un nouveau détour par le raisonnement. Dans la vie courante, on parle d'intuitions. Mes informateurs disaient souvent «sentir» que l'autre mentait, «sentir» qu'il fallait agir autrement; ils parlaient de «pressentiment» comme moteur d'une décision, surtout quand le choix des soins était une question de vie et de mort. Ainsi beaucoup de cas qui auraient pu susciter des dilemmes moraux vu de l'extérieur étaient vidés de leur sens et de leur incertitude par les acteurs, qui se fiaient à des décisions passées prises dans des circonstances similaires et qui leur avaient donné satisfaction.

Dans le cas moldave, il fallait un élément de surprise pour remettre le raisonnement en route, comme l'introduction de ces équipements de fitness tout neufs sur lesquels montaient des septuagénaires en jupes longues, têtes enserrées 
dans des foulards, quelques blouses superposées, serrés jusque sous le menton en plein été. À partir de là, il est devenu possible de se demander ce que désiraient finalement les personnes âgées? Quels étaient les soins les plus appropriés pour eux, dans le souci d'un équilibre à la fois physiologique et psychologique? La question se posait, nouvelle, devant l'adhésion de ces femmes âgées à cet élément de modernité, à cette nouveauté qui les faisait rougir comme de jeunes filles, sous l'oeil mi-amusé mi-moqueur des plus jeunes qui, précision non anodine, n'avaient pas accès à une salle de fitness eux-mêmes.

Les «intuitions» peuvent fausser le raisonnement, car elles font en sorte que l'expérience, utilisée comme argument de raison et pourtant investi d'émotion, pèse sur le nouveau raisonnement. Ce phénomène explique que, même devant le succès étonnant du fitness parmi les plus de 70 ans, les jeunes prédisaient l'abandon du centre une fois les subventions pour la nourriture terminées (leur expérience leur ayant appris que ce type de miracle ne dure pas). Cette «intuition» a de plus eue des conséquences immédiates sur la formulation de la demande de renouvellement de financement du centre. La construction de la salle continuait à être considérée comme un choix discutable de l'investisseur occidental, résultant d'une méconnaissance classique des besoins de l'Est par l'Ouest, et seul le plaisir de voir surgir une construction finalisée dans le village, objet de fierté, bénéficiait de l'adhésion de la communauté. Encore une fois, qu'en est-il de la remise en question de la conception locale du «bien-être des personnes âgées»?

\section{Les aspects expérientiels: l’approche phénoménologique}

L'approche phénoménologique, l'expérience vécue à travers son corps, la manière d'habiter son environnement, la perception intérieure et subjective de son rapport au monde ont fait l'objet d'un réel essor en anthropologie sociale depuis une dizaine d'années. L'approche phénoménologique en anthropologie ouvre un champ d'investigation important dans le domaine de la santé. Parmi les premiers à explorer cette voie, on retrouve le récit de l'habitation du temps par les malades en phase terminale d'Irving (2004) ou, dans le domaine de l'anthropologie de l'éthique, une étude de Rydstrom sur l'observation de l'éducation des jeunes filles vietnamiennes à partir de la posture du corps (2003). Jarrett Zigon (2013) va plus loin et propose l'analyse phénoménologique dans sa dimension existentielle. Sommes-nous à l'aise? Qu'est-ce qui nous «gêne»? Et dans quelle mesure cet inconfort physique et intérieur se traduit-il par une certaine prise de position morale?

Dans le cas de la révélation causée par la salle de fitness, ce qui gênait les jeunes était la jeunesse et la sexualité entrevues de leurs aînés (la cantine et la salle de fitness déclenchaient des rencontres entre les deux sexes, même si les femmes, plus 
longévives, étaient majoritaires), dans une communauté où, après un certain âge, on ne parlait plus de désir, où un veuf ou une veuve restait le plus souvent seul jusqu'à la fin de ses jours. Cela gênait d'autant plus dans une société où la jeunesse et le bien-être de beaucoup de personnes entre deux âges étaient compromis- à cause de la séparation du conjoint due à la migration, du danger conséquent de l'adultère et du manque de moyens financiers des femmes pour entretenir leur beauté. Sans parler proprement dit de jalousie envers l'accès privilégié des personnes âgées au fitness, que personne n'aurait avoué, certains villageois plus jeunes trouvaient que «c'était un peu trop».

La question qui se pose ici au chercheur est de se demander si remplacer «l'intuition» et le «pressentiment» dont parlent les acteurs par une subjectivité exprimée (quand elle parvient à s'exprimer) en terme d'inconfort moral, aide à mieux ethnographier et analyser un vécu difficilement exprimable par les acteurs et difficilement observable par l'anthropologue. «Sentir que c'est un peu trop» était l'expression récurrente formulée entre deux moues, soit de réprobation soit d'amusement, par les plus jeunes; ils résumaient ainsi leur position éthique concernant les soins aux personnes âgées. Si l'ouverture de cet espace conceptuel est un peu hasardeuse, elle permet néanmoins de prendre en considération un éventail plus large de facteurs qui entrent dans la détermination du jugement moral et de l'action.

\section{CONCLUSION}

Considérant la multiplicité de facteurs en présence, seule la richesse du récit ethnographique peut permettre d'apporter des éléments de réponse aux questions précises posées dans le domaine de l'éthique des soins. L'analyse conceptuelle, quant à elle, peut permettre d'élargir et d'enrichir l'éventail des outils d'investigation pour permettre à l'ethnographe de recueillir l'ensemble des données nécessaires pour une vue plus complète sur un phénomène. La vigilance méthodologique s'impose pour éviter l'écueil de la simplification des arguments rhétoriques puissants utilisés par défaut, dans l'absence de données précises. Toute réponse ou théorie risque de rester partielle et locale. Mais, au regard des avancées qu'elle permettrait de faire en matière d'action sur le terrain, elle n'est pas moins nécessaire. 


\section{RÉFÉRENCES BIBLIOGRAPHIQUES}

Baumard, N. et D. Sperber. 2007. «La morale», Terrain, no. 48, pp. 5-12.

Baumard, N.S. 2010. Comment nous sommes devenus moraux: Une histoire naturelle du bien et du mal, Paris: Odile Jacob.

Boltanski, L. et L. Thévenot. 1991. De la justification. Les économies de la grandeur. Paris: Gallimard.

Cross, J. et H. N. MacGregor, H. 2010. 'Knowledge, Legitimacy and Economic Practice in Informal Markets for Medicine: A Critical Review of Research', Social Science and Medicine 71.9:1593-1600.

Dunn, E. C. 2004. Privatizing Poland: Baby Food, Big Business and the Remaking of Labor. Ithaca/Cornell University Press.

Fabian, J. 1983. Time and the Other: How Anthropology Makes Its Object. Columbia University Press.

Festinger, L., H. W. Riecken et S. Schachter. 1956. When Prophecy Fails: A Social and Psychological Study of a Modern Group that Predicted the Destruction of the World, University of Minnesota Press.

Firlit, E. et J. Chlopencki. 1992.- «When Theft is Not Theft». In Wedel, J. ed. The Unplanned Society: Poland during and after communism. NY: Columbia University Press.

Heintz, M. 2006. "Be European, recycle yourself!»: The changing work ethic in Romania. Muenster: LIT Verlag.

Heintz, M. 2009. Ed. The Anthropology of Moralities. Oxford: Berghahn.

Herzfeld, M. 1987. Anthropology Through the Looking Glass. Critical Ethnography in the Margins of Europe. Cambridge: CUP.

Howell, S. 2007. The Kinning of Foreigners. Oxford: Berghahn.

Irving, A. 2004. «Life Made Strange: An Essay on the Reinhabitation of Bodies and Landscapes. » In Qualities of Time. ASA Monograph Vol 41, ed. Wendy James and David Mills, 317-330. Berg: Oxford and New York.

Kahneman, D. 2011. Thinking, Fast and Slow. NY: Farrar, Straus and Giroux.

Lambek, M. 2010. ed. Ordinary Ethics: Anthropology, Language, and Action. Fordham University Press.

Mahmood, Saba. 2005. Politics of Piety: The Islamic Revival and the Feminist Subject. Princeton: Princeton University Press. 
Robbins, J. 2004. Becoming Sinners: Christianity and Moral Torment in a Papua New Guinea Society. Berkeley: University of California Press.

Rydstrom, H. 2003. Embodying Morality. Honolulu: Hawai'i University Press.

Scheper-Hughes, N. 1995. «The Primacy of the Ethical: Propositions for a Militant Anthropology. » Current Anthropology 36 (3) (June): 409-20.

The Executive Board of the American Anthropological Association. 1947. Statement on Human Rights. American Anthropologist New Series, Vol. 49, No. 4: 1, pp. 539543.

Wikan, U. 2002. Generous Betrayal: Politics of Culture in the New Europe. University of Chicago Press.

Wikan, U. 2008. In Honor of Fadime: Murder and Shame. Chicago: University of Chicago Press.

Zigon, J. 2013. «On Love: remaking moral subjectivities in post-rehabilitation Russia». American Ethnologist 40 (1): 201-215.

\section{Pour prolonger la réflexion:}

Fassin, D. 2012. Ed. A Companion to Moral Anthropology. Malden, MA: Wiley-Blackwell.

Lambek, M. 2010. Ed. Ordinary Ethics: Anthropology, Language, and Action. Fordham University Press. 